DOI: http://dx.doi.org/10.4314/ljh.v27i2.2

\title{
The Ecology of Gahu: Participatory Music and Health Benefits of Ewe Performance in a Canadian Drum and Dance Ensemble
}

\author{
Kathy Armstrong \\ Adjunct Faculty, Department of Music, Institute for African Studies \\ Carleton University, Ottawa, Canada \\ kathy.armstrong@ carleton.ca
}

Received: April 29, 2016 / Accepted: September 12, 2016 / Published: December 16, 2016

\begin{abstract}
Ghanaian music and dance provide a rich environment for social interaction, which is a significant contributory factor to health and well-being, both for individuals and the communities in which they live. The vibrant and energetic drumming and dance of the popular Ewe piece Gahu offer numerous opportunities for participatory music-making, not only in Ghana but throughout the world, in performance, educational and community settings. Through video analysis and discussion of cross-disciplinary research, this article identifies the ecological factors present in a Canadian university performance of Gahu that play a positive role in the health of the students involved.
\end{abstract}

Keywords: Gahu performance, dancing, health, entrainment, participatory music

Ghanaian recreational music and dance are a source of inspiration and pleasure to many, drawing Africans and non-Africans alike into the inclusive, full-body, artistic experience that both of them provide. The compelling sounds of interlocking rhythms on drums led by circular patterns on iron bells, supported by the perpetual motion of dancers and group singing, combine in a manner that has both an invigorating energy and timeless appeal. Factors such as the makeup of the individuals creating the music, the social relationships between them, and the space and time of the event, are instrumental in creating an environment conducive to improving social, mental and physical health. In examining links between music and wellbeing, it is useful to reflect on information from several disciplines for connections and insight. In the introduction to The Oxford Handbook of Medical Ethnomusicology, Benjamin Koen and his colleagues acknowledge the complex ways in which music and healing are intimately related to our "biological, psychological, social, emotional and spiritual domains of life, all of which frame our experiences, beliefs, and understandings of health and healing, illness and disease, and life and death" (2008, p. 4). Collaborations between researchers and scholars across disciplines are contributing to a wider pool of knowledge. These disciplines include the physical and social sciences, medicine, music and healing arts. Drawing upon research from these fields, as well as a discussion of musicking, participatory musics and entrainment, this article will examine the ecological factors present in a Canadian student performance of Ghanaian drumming and dancing that provide an environment conducive to positive health.

Ecology deals with relationships and interactions among individuals. These interactions are what Christopher Small (1998) describes in his concept of "musicking". He writes, "if we 
widen the circle of our attention to take in the entire set of relationships that constitutes a performance, we shall see that music's primary meanings are not individual at all but social" (p. 8). This kind of social interaction is a significant contributory factor to health and wellbeing, both for individuals and the communities in which they live, as Clift and Hancox (2010), Putnam (2000), and Stige (2006) have shown. Research conducted with choirs clearly documents the health advantages of singing in a group setting. Advantages include increased positive mood, focused concentration, controlled deep breathing leading to reduced anxiety and stress mitigation, improved immune system, social support, and cognitive stimulation (Clift \& Hancox, 2010). Although both passive and active music-making have been shown to provide health benefits to individuals in clinical therapeutic settings (Bernatsky, Strickner, Presch, Wendtner, $\&$ Kullich, 2012), the environment of those engaged in the music-making including the interactions and relationships among people, are highly important and can amplify the benefits of music. In therapeutic fields, positive impacts on health and wellness have been shown when the focus moves from the individual participation of the client to include interaction between the client and the therapist, and in some cases a larger community (Stige, 2006).

The importance of relationships and community are also reflected in the participatory nature of music itself. An ensemble of Ghanaian drumming, singing and dancing encompasses many of the elements found in participatory musics as outlined by Thomas Turino (2008). These large social and musical gatherings facilitate multiple layers of skill, experience, and intent in the music-making, often emphasising participation over artistic output. Many participatory musics use rhythm as a central unifying factor (Turino, 2008). Recent research in science and medicine supports the premise that rhythm plays an important role in wellness and the promotion of healing (Thaut, 2013; Clayton, Sager, \& Will, 2004). The concept of rhythmic entrainment is important to the discussion of music and health connections. Ethnomusicologist Martin Clayton has been examining entrainment and its application to music. He describes the concept as "the process by which independent rhythmical systems interact with each other" (2012, p. 49). The gradual process of entrainment such as between drummers and dancers in a Ghanaian-style ensemble, brings the rhythms and movements into a "consistent relationship that continues to be negotiated on an on-going basis, providing an intimate connection between participants" (Bluedorn, quoted in Clayton et al., 2004, p. 10). The factors outlined above - the participants; the space and time of the event; the relationships among those involved; rhythmic entrainment; the participatory nature of the music - have a significant impact on the health and wellbeing of members in a Ghanaian-style drum and dance ensemble. In this article, the ecological factors identified will be further discussed in relation to music and dance.

The focus of the present investigation is an analysis of the Ewe drum and dance piece Gahu, as it was performed on 23 November 2012 in the Kailash Mital Theatre at Carleton University in Ottawa, Canada, by two student ensembles that I direct - the Carleton University West African Rhythm Ensemble (WARE) and the Baobab Youth Performers. ${ }^{1}$ Gahu is a recreational piece of the Ewe people of south-eastern Ghana. A rich texture of stick drumming, dancing and singing, Gahu developed among the Ewes in the 1950's, and according to Kobla Ladzekpo, "was brought to Ghana by Yoruba speakers from Benin and Nigeria as a form of

1 The full video for this performance can be found at http://www.youtube.com/ watch?v=WLWNUdn2D6E and select stills, photos and thick description are provided in this article. Time codes will be indicated in brackets (e.g., 1:01). The video analysis described in this article provided preliminary data for the quantitative and qual itative research I subsequently undertook with the Carleton West African Rhythm Ensemble for my MA thesis (Armstrong, 2016). 
satirical commentary on modernization in Africa" (quoted in Locke, 1987, p. 5). The Gahu instrumental ensemble usually consists of gankogui (double iron bell), axatse (gourd rattles), and three supporting drums-sogo, kidi, and kagan - that are played with sticks, built in the shape of wooden barrels, with antelope skin heads and represent a range of sizes: large, medium, and small. These five supporting parts form a dense fabric overtop which the lead drummer plays on the large gboba drum, also a barrel-style drum that is played with sticks. In some instances, the tall atsimevu lead drum is used for certain sections of the piece. The gboba periodically calls rhythmic variations to which the sogo and kidi respond. Commonly, in Gahu performance, the dancers form a circle around the drums, and travel as a pulsing, rhythmic unit, and also respond to calls from the lead drum with corresponding pre-determined moves. In a Ghanaian village-style Gahu, the drum variations and choreography may be relatively simple, whereas cultural troupes in schools and urban centres often have more change and complexity in their arrangements, typical of presentational styles. Songs occur throughout, led by different singers in call-and-response style, most often in the Ewe language, with some Yoruba lyrics present from Gahu's origins. Participants enjoy the high-spirited and playful elements of the music and dance. These vibrant musical characteristics have made Gahu a popular artistic export and it is taught and performed in a variety of settings throughout the world, including many educational institutions (Dor, 2014; Locke, 1987 \& 2004).

\section{Participants}

The West African Rhythm Ensemble (WARE) is one of several performance groups available to the students in Carleton University's Music Department, which resides in the School for Studies in Art and Culture. The ensemble is comprised primarily of undergraduate music students, most of whom have never played percussion or danced in this style before. WARE, along with ensembles in other genres, contributes to the overall musical education of students enrolled in the Bachelor of Music degree program. Baobab Youth Performers ensemble is an educational afterschool arts program for teens from across the Ottawa region that employs West African rhythms, songs, and movement as the impetus for community connection. Leadership skills are developed through mentoring opportunities and public performances. Similar to WARE, Baobab Youth members generally have no prior experience with this music and dance before joining the group. As director of both ensembles, I bring them together occasionally for collaborative events and performances, as it provides a rich environment for community connection, multi-age groupings, leadership and mentoring opportunities, as well as, an economical means of working with visiting Ghanaian guest artists. The guest artist for this performance was Nani Agbeli, a dynamic and highly skilled performer in traditional Ghanaian drum and dance styles. At the time of the performance Nani Agbeli was director of Tuft University's Kiniwe Ensemble in Medford, Massachusetts. He is currently the Director of West African Music, Dance and Arts at the California Institute of the Arts. Coming from the Ewe village of Kopeyia, near the Ghanaian border with Togo, Nani grew up in a family of drummers, inspired and taught by his father, the late Godwin Agbeli, a well-respected performer, teacher and researcher of Ewe music and dance. Nani's relative youth, magnetism, high standards, and excellent teaching skills were inspiring for the students in the two Canadian groups.

My own involvement with this style of music dates back to 1984, when I was first introduced to Ghanaian rhythms, as part of my undergraduate studies, as a percussionist at the University of Toronto. In our sessions, the drumming was not accompanied by dancing or singing, yet the transformative nature of the rhythms was clearly evident and compelled me to look 
further into the music and dance. In addition to the percussion class at the University, I participated in workshops and later joined a community ensemble whose members were both Canadian and Ghanaian-born. Eventually I travelled to Ghana in 1990 for several months of immersive study. Through subsequent years of studying in Ghana, and teaching and performing the music and dance in diverse settings, my appreciation of the transformative power of this performance has only grown as I have observed a multitude of health benefits in those participating.

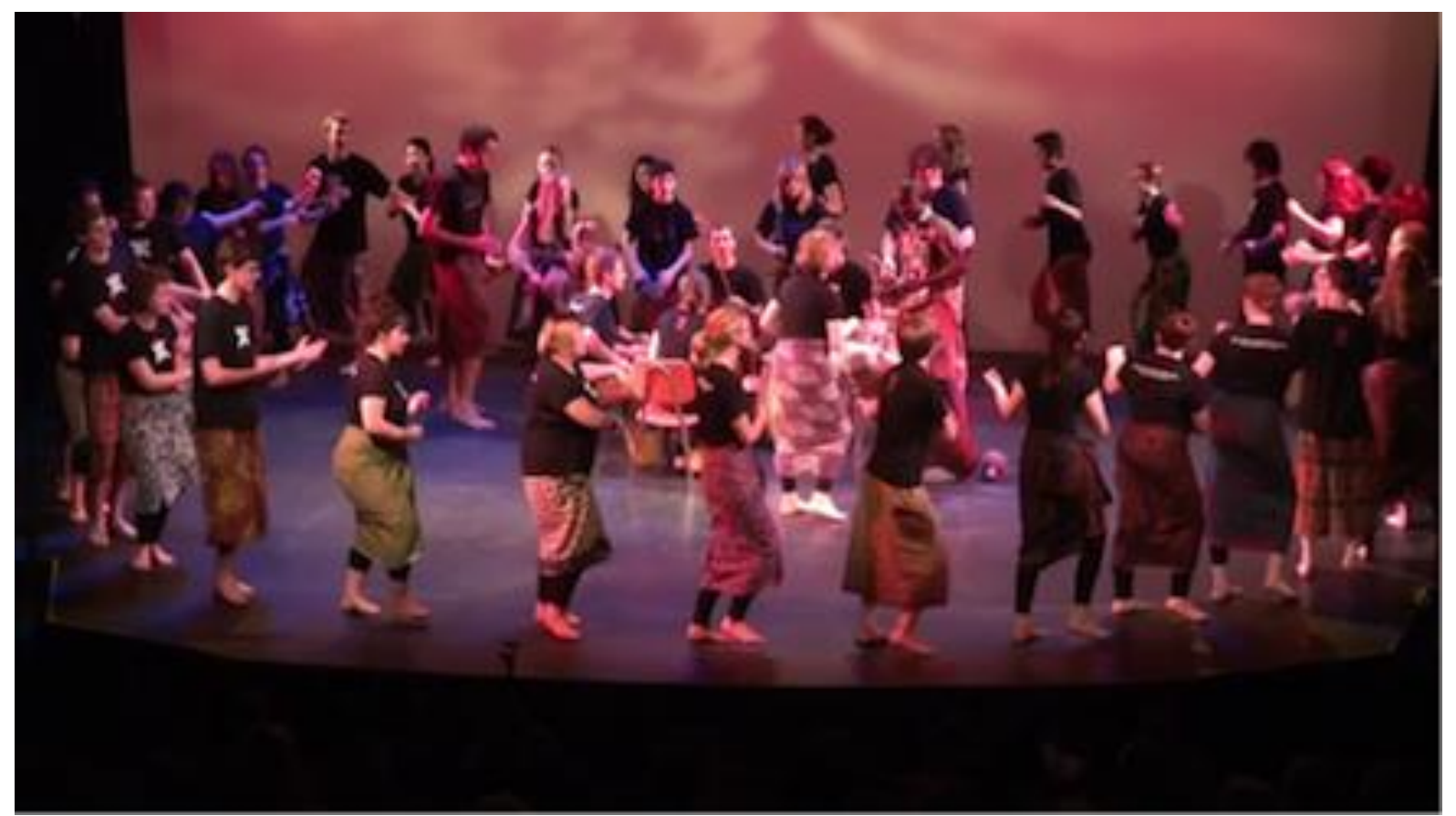

Figure 1: Still (1:01) from the recording of a Gahu performance on 23 November 2012 at Carleton University, Ottawa, Canada, by WARE and Baobab Youth Performers.

Ethnomusicologist Steven Friedson (2009) has said that Ghanaian Ewe music "does more than expend energy - it makes it!" (p. 117). Just what is it about this music and dance that creates such extraordinary energy? Figure 1 shows the first video still (1:01) of the Gahu performance on 23 November 2012 at Carleton University. This Canadian setting is a formal stage in a hall that seats approximately 450 audience members and has professional lighting and sound capabilities. The performers are arranged in a circle, with the drums in the center, radiating sound and energy out to the dancers and back again. This typical Gahu formation is often seen in Ghana. Performers are a self-contained unit, engaging primarily with each other and not so much with the audience, as is otherwise common on a stage such as this. In this performance, there were approximately 45 people involved, generally moving and playing together. Occasionally, an individual performer may catch the attention of the audience for various reasons: perhaps a louder singing voice, foot movements that might be slightly out of sync, particularly integrated movements, and so on. The basic dance movement involves advancing in the circle by taking two steps with each foot, alternating right, right, left, left, and with the weight on the back leg which is held straight, thereby producing a subtle and stylistically desirable hip movement. The upper body leans forward slightly and the arms sway in tandem across the body, swinging in the opposite direction of the front foot. These movements produce a balanced effect 
that is both grounded and forward-moving. It is clear though, that not everyone moves at precisely the same time, particularly at the beginning of the piece (1:02). Concepts of groove, synchronicity and entrainment will be discussed in the course of this article. Some call-andresponse singing in the Ewe language is heard on top of the gankogui and axatse, and soon the supporting sogo, kidi and kagan drum parts begin with their interlocking patterns (1:02). When the lead drummer plays the first variation call on gboba, the dancers signal acknowledgement by raising their arms before bending low in a more vigorous move (1:03). The tempo then increases, guided by the gboba, and the group starts to cohere their movements to a greater extent. The lead drummer calls the dancers to exit the variation and we see them respond by returning to the basic move, with noticeably more energy and connection to one another than they had exhibited at the opening of the piece.

\section{Relationship}

Christopher Small (1998) views a musical event as an encounter between human beings, with relationship at the heart of the meaning. Having introduced the participants in this performance, let us now consider Small's question: "What does it mean when this performance takes place at this time, in this place, with these participants?" (1998, p. 10). This is a critical question and one that is central to understanding the health value of the performance of Gahu. For members of WARE and Baobab Youth, Gahu is a foundational piece in their repertoire, one that is performed every season, along with other styles of Ghanaian recreational music. The repetition of Gahu every year provides returning members of the groups with familiarity while, at the same time, offering newcomers a well-honed structure in which they can learn new skills and socially connect with other members. With many options for drum and dance variations as well as songs, Gahu can be experienced as both the same and new to those participating. In my role as an educator and director of these ensembles, I look for ways to challenge individual students by having them change roles in pieces every year. This provides an experience of 'flow' (Csikszentmihalyi, 1990) for the participants, important in the discussion of the links between music and wellbeing.

Csikszentmihalyi states that when there is an absence of stress, combined with a balance between challenge and skill level, participants can experience flow, leading to feelings of pleasure, focus and motivation (1990). The experience of Gahu is different every time, due to the composition of the group, the setting, the emotional and physical engagement of the participants, and the overall energy and flow generated by the experience. I can say from my own perspective, having led and participated in hundreds of performances of Gahu over three decades, that no two events are alike. This addresses the question Christopher Small poses above (this performance, this time, this place, these participants) as it is the subtle and not-so-subtle differences in each performance that contribute to Gahu being such an engaging and enriching experience. Being in-the-moment is crucial to this style of music. The nature and meaning of the experience lie in the actions of people. Performing on stage can produce feelings of nervousness and anxiety that may inhibit the focus on the present moment. However, the style of music and the abundance of participatory factors encourage the students in this performance to deeply engage with the music, one another, and the energy of the guest artist. Both Baobab Youth and WARE are accustomed to performing participatory styles in various presentational settings. In my teaching, I try to bring certain fundamental qualities of a Ghanaian Gahu experience to my students, while acknowledging our outsider efforts - the diverse individual cul- 
tural experiences (or backgrounds) and norms that performers inevitably bring into the performance. Many of those fundamental qualities I consider important in my teaching are found in characteristics of participatory musics, which I will discuss later in this article.

Small (1998) highlights not only the relationship between sounds, but also the participants' relationship to the outside world, observing that musicking is a way of engaging in the relationships between relationships, which, in his opinion, is where the meaning lies (p. 48). For instance, in this performance of Gahu, we do not only see the establishment of musical and social relationships among the participants, as well as, between them and the audience, but everyone brings in their relationship with the outside world, thereby providing meaning based on their own projections. This could include feelings of belonging or exclusion, concepts of education and music or experiences related to Africa and the community. Musicking is a way of knowing our own world through the experiential order of relationships. "In knowing it, we learn how to live well in it" (Small, 1998, p. 50). For students in the formative adolescent and young adult years, participating in Gahu provides a framework within which to explore their identity in relation to others.

In addition to the relationships between the participants, one of the most important components of this analysis is the concept of participation itself. A definition of the term that resonates with this study comes from music therapist Brynjulf Stige (2006):

Participation is a process of communal experience and mutual recognition, where individuals collaborate in a socially and culturally organized structure (a community), create goods indigenous to this structure, develop relationships to the activities, artefacts, agents, arenas and agendas involved, and negotiate on values that may reproduce or transform the community. (p. 134, emphasis in original)

Stige's definition reflects his experience and research in community music therapy sessions, underlining the idea that musical interventions for promoting health involve a series of relationships, and not only the individual participation of the client or patient. In this way, participation becomes more than the acquisition of knowledge and skills; it is about becoming part of an evolving community (2006, p. 127). This reflects the kinds of experiences members of WARE and Baobab Youth Performers have, as they form a community while obtaining new skills and working on a collaborative activity. Participation in this kind of drum and dance ensemble provides many opportunities for bodily knowledge and social interaction: interlocking drum patterns that "speak" to one other, musical dialogue between the lead drum and dancers, full body engagement with rhythm, active listening ${ }^{2}$ and non-verbal communication. Each of the groups in this video performance have navigated these musical and personal relationships within their respective groups in the context of a positive educational environment. There was a mutual recognition when WARE and Baobab Youth met to work together in preparation for this particular concert collaboration. Their prior experience with this music and dance along

\footnotetext{
${ }^{2}$ Active listening refers to a way of listening that requires full concentration and attentiveness. A term used in therapy and conflict management fields, it promotes deeper understanding between people. Learning how to listen for and respond to complex drum patterns develops a similar kind of active listening. My students have remarked on their increased ability to focus, to absorb content in academic lectures, and to interact socially and musically with peers as a result of their engagement with Ghanaian drum and dance repertoire (Armstrong, 2016).
} 
with my teaching style provided an environment that encouraged certain values and expectations such as inclusivity, social connection, and the freedom to make mistakes and to be themselves, collaborative effort, and personal challenge, including the acquisition of new skills (Armstrong, 2016). Joining together for this larger concert allowed for connection to a wider community, fostering personal enjoyment and a positive educational environment.

\section{Participatory Music-Making}

In his book Music as Social Life, Thomas Turino (2008) observes that in participatory music-making throughout the world, there is often no distinction between audience and performer, and that everyone present can and should participate, to the best of their ability (p. 29). This reinforces a feeling of belonging, which has strong social health implications (Centre for Addiction and Mental Health et al., 2013). The primary focus is on the doing, or musicking (Small, 1998, p. 8). The social factor of the event is valued over the quality of the sound (or in the case of Gahu, sound, and movement). In his research, Turino (2008) has identified some features and practices that are frequently found in participatory musics throughout the world. They are important to our understanding of the connections between health and Ghanaian music and dancing. These features both inspire and support participation and contribute to social cohesion, which has been shown to increase health and wellness (Stige, 2006; Clayton et al., 2004). We shall consider four of Turino's (2008) characteristics of participatory music-making here, beginning with form and repetition.

The form of participatory music is usually open and depends on contributions from the individuals participating. The musical events are not fully pre-planned, and embody many inthe-moment decisions. The music contains cyclical patterns and ostinati, and the form is often made up of short sections. The constancy of these sections provides security for the performers; repetition might be boring for an audience but for the participants it actually heightens the intensity of the experience.

Rhythmic repetition and social synchrony: These important features of participatory music-making include repetition and mirroring, in both gesture and body language. This occurs in dance and music and provides a level of comfort for the participants. It is a tacit identification between people that helps them feel connected and successful in their endeavor. These musical features become signposts and markers since the music is not scripted. This results in a felt sense of belonging.

Musical texture, timbre, tuning, and density: Some shared musical elements contribute to participatory musics throughout the world. These traits inspire participation without too much worry that an individual will be singled out. Elements such as a dense texture, overlapping parts and buzzing sounds on the instruments, all provide a cloaking function. In addition, Turino notes that the acceptable tuning of instruments and voices is "wider" in these styles, again allowing for maximum participation.

Virtuosity and soloing: One might be under the impression that music in participatory cultures does not allow for exceptional talents to be seen and heard. This is not the case. Virtuosity is certainly present and valued, however it is usually situated within the texture of the larger experience, otherwise it would discourage participation. Often one can see examples of call-and-response that allow for brief soloing and virtuosity. This also appears in dance forms, when one dancer takes to the floor in the center of a circle for a brief time before the next soloist. This sequential (and sometimes simultaneous) soloing is common. 
With these characteristics in mind, one can state that Gahu offers an ideal artistic experience for Canadian students, inviting them into a community of social music-making. If we apply Turino's participatory framework, we see that the musical form of Gahu is open, led by the large gboba drum and framed by the cyclical gankogui pattern. The texture is dense, with several overlapping and interlocking stick drum patterns as well as a few axatse rattles providing a buzzing rhythmic background. There are many examples of observable gestural mirroring among the drummers, dancers, and between these two performing groups.

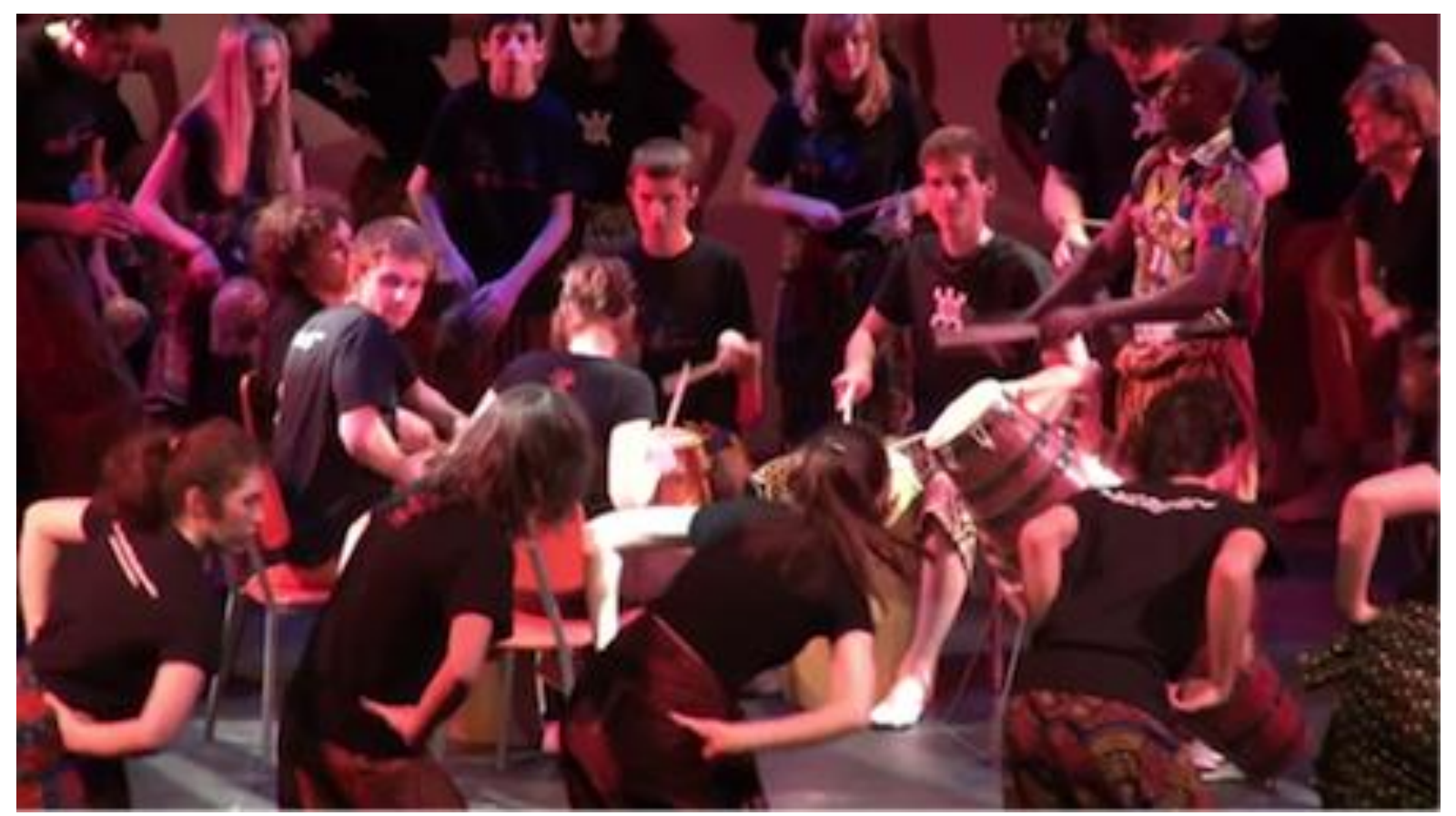

Figure 2: Still (1:03:30) from Gahu performance on 23 November 2012.

This mirroring can be seen in the visual examples provided. Several Ghanaian styles of music and dance support multiple levels of skill and experience, and Gahu is no exception. Participants in the particular performance under consideration here encompassed a range of ages, as well as a variety of musical and non-musical backgrounds, rhythmic skills, comfort with movement, and experience with the style of music, allowing for mentoring opportunities during rehearsals and performances. With a basic competence, the groups could work with a very gifted guest artist, in this case, Nani Agbeli, who particularly stood out in this student performance, while soloing on top of the texture of rhythm and dance. In the second video still in Figure 2 we can see some of the participatory factors and relationships present.

At this moment in the performance (1:03:30), the lead drummer calls the second variation in this Gahu. The sogo and kidi supporting drums respond with a rhythmic answer. This changes the sound of the drumming texture and puts the constant pattern of the kagan drum into a new rhythmic relationship with the other instruments. The dancers also respond to the call by placing their hands on their hips and leaning forward, while continuing to move forward with the double foot step. Part two of this variation sends the dancers even lower, accentuating the uniformity of their movements. One of the drummers turns his head and expresses his appreciation of this new energy, making eye contact with some of the dancers. It is apparent that the group's cohesiveness has increased during this second variation due to the intensity and synchrony of movement and sound, and participants' engaged facial expressions. Once again, 
the lead drummer calls the dancers and drummers to exit the variation and return to the basic patterns, noted by the synchrony of the dancers and increased rhythmic consistency among the drummers.

\title{
Rhythmic Entrainment
}

Beyond the rhythmic synchrony we hear and observe between dancers and drummers, additional activity is occurring at a deeper physical and neurological level. New research into the functioning of the brain and body has provided us with a scientific understanding of the power that rhythm has to unite people and provide health benefits. In his book, Rhythm, Music and the Brain, Michael Thaut (2013) observes the recent shift in the music therapy paradigm, from a concept rooted in the social sciences to one that is informed by neuroscience (p. 113). We know that music plays an important function in bringing people together; it provides pleasure, and produces feelings of wellbeing. Throughout history, music has been considered a useful tool for developing emotional expression, providing a distraction from pain and suffering, increasing social interaction and building community (LaGasse \& Thaut, 2012, p. 153). Thaut notes that music is found in all known cultures throughout history, which indicates that it may play more than an accompanying role in our biological and neurological functions. He writes:

\begin{abstract}
Brain research involving music has shown that music has a distinct influence on the brain by stimulating physiologically complex cognitive, affective, and sensorimotor processes. The fascinating consequence of this research for music therapy has been a new body of neuroscientific research that shows effective uses of music with therapeutic outcomes that are considerably stronger and more specific than those produced within the general concept of well-being. (2013, p. 115)
\end{abstract}

As I mentioned in my introduction, the concept of entrainment is central to our understanding of music and the brain, as well as to the present analysis. All organisms have the capacity to respond to external rhythm, such as patterns of day and night, weather, and tides, and these are necessary evolutionary tools. As humans, we have the added skill of being able to entrain to a wide variety of tempi, integrate what we hear into our minds and bodies, and then generate rhythmic responses to what we hear (Philips-Silver, Aktipis, \& Bryant, 2010). This skill is important for communication, attracting individuals for mating, and gathering in groups. The integration of both the perception and production of rhythm makes it possible for humans to systematically alter rhythmic production, based on the rhythm we perceive in our environment (Philips-Silver et al., 2010). This enables us to achieve group synchrony, or social entrainment. Ethnomusicologists Will and Turow (2011) note that although the terms are sometimes used interchangeably in the literature, synchronization does not necessarily indicate that entrainment is taking place. Entrainment occurs when two or more autonomous rhythmic processes become more aligned over time, as a result of prolonged interaction. This means that they each must have an internal energy source that is capable of producing rhythm on its own. Resonance, for instance is not considered a form of entrainment (Will \& Turow 2011, pp. 1112).

For members of WARE and Baobab Youth Performers, the entrainment between drummers and dancers that evolves over the course of a 20-30 minute interpretation of Gahu is palpable. Entrainment is occurring at both the intra-individual and intra-group levels (Clayton, 
2012, p. 51). Each performer is entraining within themselves and also within the group. After a prolonged period of interaction, the drummers have a tighter groove, and the dancers' bodies are much more aligned with the drum rhythms and each other. Playing music together, dancing, and singing in a group are all forms of social and musical entrainment, but these are not only recreational pleasures. Entrainment has been studied in both biological and mechanical systems (Clayton, 2012, p. 49). As a form of social synchrony among humans, entrainment promotes cooperation and affiliation, which can facilitate higher-level organization that needs real-time processing of rhythmic information such as large-scale building projects, collective foraging and predation. The ability of a community to drum and dance together can make these processes more efficient and effective (Philips-Silver et al., 2010). Clayton et al. (2004) have been examining the implications of entrainment in music research. Looking at verbal and non-verbal signals, which can also be understood as language and gesture, they highlight the process of entrainment and the importance of the shifting yet consistent relationship that happens. They also observe that "entrainment appears to be one of the fundamental processes providing an intimate connection between individuals, others, and the world around them" (p. 16). In fact, research shows a connection between entrainment and positive affect in communities (Warner quoted in Clayton et al. 2004, p. 13).

Interestingly, "rigid entrainment and precise synchrony in human rhythmic processes are not necessarily associated with health or positive affect. Entrainment that is not too 'perfect' generally provides a more positive social experience" (Clayton et al. 2004, 13). This supports Turino's (2008) notion that participatory musics inspire people to join in, where a high level of synchronization is not required, and where social enjoyment is paramount. My involvement with Ghanaian music and dance as a student, educator and performer has provided experiences in both professional and community ensemble settings. In these situations - the social synchrony that develops over time, along with the musical entrainment between participants and instruments - combine for highly pleasurable encounters. The energy is often electric and palpable and there is an enjoyment and delight in sharing the experience with others, musicking together with a common intention.

Groove is a concept that often comes up in discussions of rhythmic styles of music. In Music Grooves, Keil (1994, p. 96) states that "the power of music lies in its participatory discrepancies. Music, to be personally involving and socially valuable, must be 'out of time' and 'out of tune"'. This suggests a kind of entrainment between instruments and people that is not too tightly aligned but remains in a consistent relationship. These traits are found in many styles of music, including the Gahu performance I am examining. The variable rhythmic synchrony along with the slightly wider tuning of some of the singing from the students is combined together in an experience which is powerful, personally involving, and valuable to the students in terms of their social, mental, and physical health (Armstrong, 2016). One of Keil's alternate descriptions of participatory discrepancy is a "semi-conscious or unconscious slightly out of syncness" (1994, p. 96). Participants in this Canadian performance of Gahu move in and out of the collective groove depending on their skill set, their level of comfort with the musical material and their enjoyment in the moment. Their complete focus on what they are doing can be observed. How synchronized they are within themselves or in relation to the larger group is something they may be consciously aware of, or perhaps that knowledge resides deeper, at a felt-sense level.

This felt relationship to rhythm is something James Burns (2010) has written about in detail. His theoretical approach to analyzing several styles of African music recognizes a multi- 
sensorial perception where the rhythmic background of clapping, dancing and singing is integrated into the understanding of the instrumental rhythms that occur. His concept of rhythmic archetypes suggest a whole-body perception of Ghanaian music. Burns describes these archetypes as rhythmic patterns that exist both on the "surface-structural level and the deep-structural level" (Burns, 2010). They exist in relation to a "shared rhythmic background" and performers visualize and feel the timing rather than count pulses. He argues that the "rhythmic archetypes produce a tactile image that becomes embedded into the tactile memory over time, creating both a physical and mental impression of each rhythm" (Burns, 2010). I have observed this with my university students who can competently play the rhythm of the sogo drum in Gahu yet often cannot place it correctly within the timing of the whole piece. Where it lies in the gankogui cycle, and the combination of open (bounced) and closed (muted) sounds on the drumhead compels the player to develop a physical relationship with the part, one that is lodged in the larger texture of rhythms and movements in order to play it correctly. This example highlights the complex elements involved in playing Ghanaian rhythms, particularly the importance of the relationship and entrainment occurring between rhythmic elements. Two additional Gahu examples will illustrate these points.

In the photograph in Figure 3, the lead drummer has called for another variation, one that begins with the dancers' arms raised, as well as a vocal response to his spontaneous vocal call (WARE, 2012, 1:06:15). The energy level all around is clearly heightened and the music reflects it with increased tempo and additional synchrony among dancers. There is also more fluidity in the hand motions of the supporting drummers. This two-part variation then shifts to a section that sees the dancers engage in a weave-like movement, face-to-face, as they move around the circle in opposing directions, meeting and visually greeting other members of the ensemble. For some, even though they have practiced the dance movements, there is an element of discomfort, as their age and Canadian lifestyle rarely calls for this kind of intimate interaction through the arts. Also, these two groups do not know each other that well socially since they do not often perform together. However, most are observed making some kind of visible connection when passing other participants. Even those who may feel somewhat awkward or inexperienced are responding to gestures from others who are more relaxed and confident in their performing roles. An audible "whoop" is heard when the lead drummer delivers them back to their original position with a cue to end the variation, showing pleasure in completing the movement. 


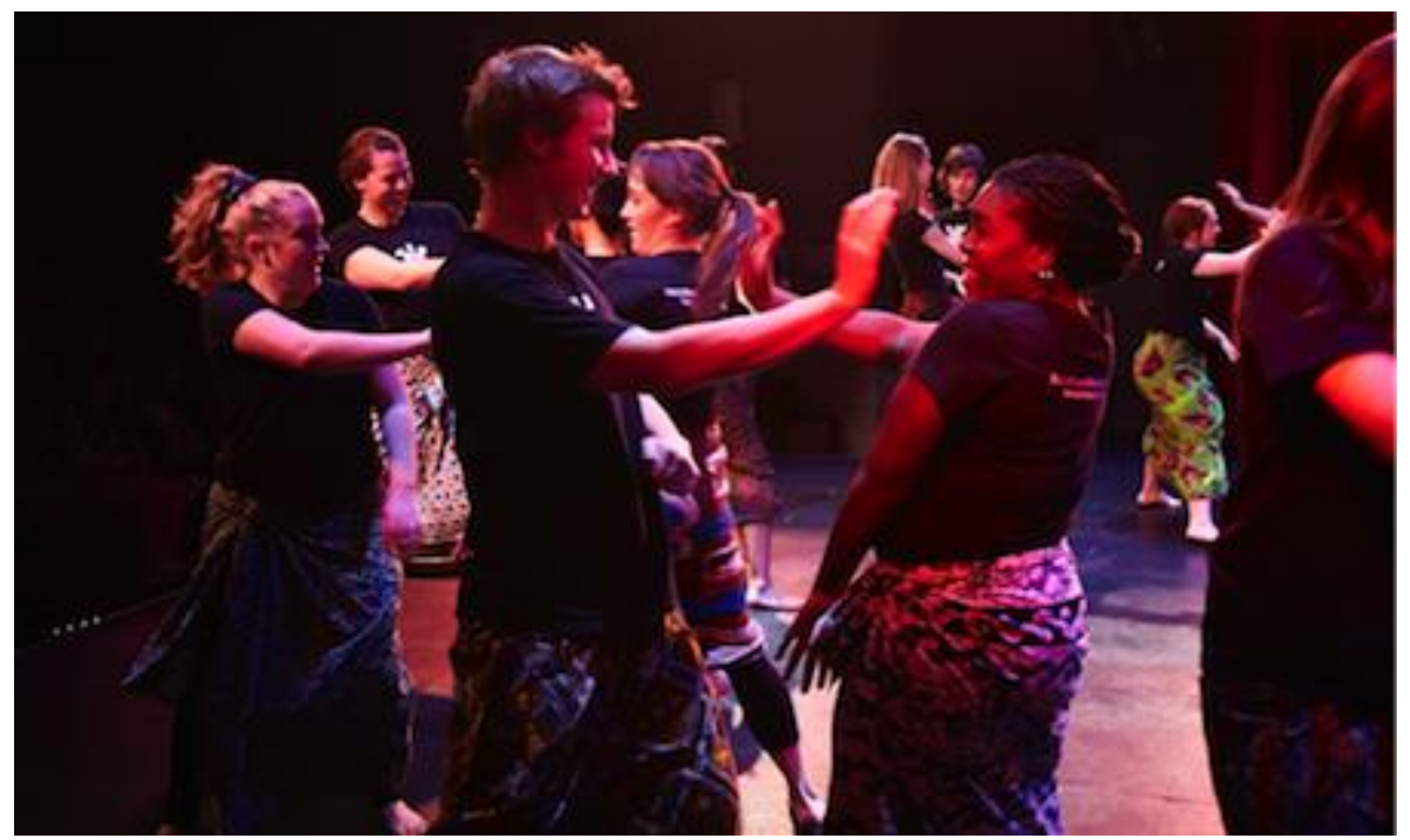

Figure 3: Photograph of Gahu performance (photo credit: Lyndon Goveas)

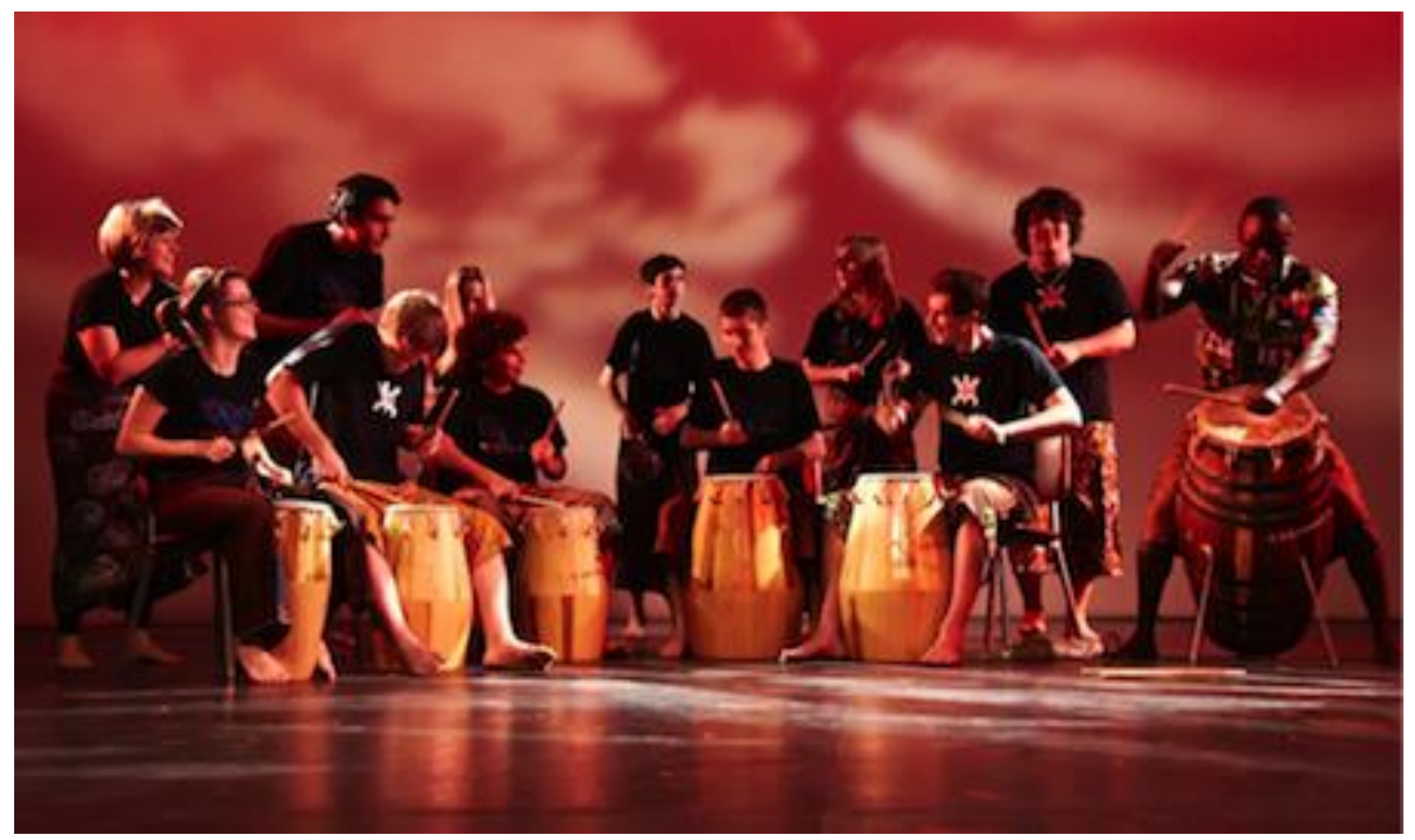

Figure 4: Photograph of Gahu performance (photo credit: Lyndon Goveas)

In the picture in Figure 4, the dancers have exited the stage while the drummers rearrange chairs for the guest artist, Nani Agbeli, to perform his solo on the gboba. Initiated by Nani, this move allows for the finale to be more presentational in style, taking advantage of the large stage and turning to communicate with the audience (WARE, 2012, 1:13:00). It is still within the parameters of participatory music-making, allowing for the student drummers to be part of the highly-skilled solo demonstration. It challenges them to the edge of their physical 
limits, where they are fortified by the energy of the soloist. The dancers can be heard offstage expressing themselves vocally, connecting to the final display.

Describing a model of social entrainment, McGrath and Kelly suggest that during musicking there is a "profound association between different humans at both a physiological and physical level" (cited in Clayton et al., 2004, p. 10) and this contributes to a sense of identity and belonging. Belonging to a community facilitates empowerment and social capital, which are major determinants of health (Freeman, King, \& Pickett, 2011; Stige, 2006). In Western approaches to therapeutic health research, the focus is often on an individual's participation and experience. Stige (2006) advocates for a model that assumes there is more to participation than "being there" or "joining in" and that a collaborative approach is desired, one that effects change in all parties (pp. 122-123). This therapy model suggests that collaborative music-making, as in the case of Gahu, has a strong potential for providing positive health benefits. The relationships and experiences that come from participatory music-making allow us to accumulate social capital.

The paradox of our contemporary world is that through technology we are able to connect to almost any person and place we desire, at any time, and yet often we find ourselves becoming more isolated and shut away from one another. This contradiction can be overwhelming to many, producing feelings of stress and anxiety, as well as deeper mental, social and physical health problems (Armstrong, 2016; Centre for Addiction and Mental Health et al., 2013). It is clear from emerging research in several disciplines, that being in relationship with other humans is central to our health and wellbeing.

As mentioned in the introduction, connections between singing, and health and wellbeing have been well documented in past studies. In their international study of community-based choirs, Clift and Hancox (2010) employed the World Health Organization's Quality of Life questionnaire, which measures physical health, psychological health, social relationships, and environment, in the context of a person's culture and value systems, and in relation to their personal goals, standards, and concerns. This type of research would be valuable to undertake with participants in a Ghanaian-style drum and dance ensemble and one could project that similar results would be generated from these kinds of physiological studies and survey interviews.

From the preceding visual examples, descriptions and discussion, we have observed several ecological elements in the performance of Gahu that are contributing to the health and wellbeing of the students: composition of the participants and the relationship they build with one another, the space and time of the event, multiple characteristics of participatory musicmaking, moderate rhythmic entrainment in both the drumming and dancing, and social cohesion. These factors are all at play within a framework of participatory musicking. Participating in a Ghanaian-style drum and dance ensemble offers not only unique learning and social opportunities for those involved, particularly for these students, but a way of being in relationship with other individuals that facilitates positive mental, social, and physical health advantages. The patterns found in the drumming, dancing, and singing of Gahu, combined with the nonverbal gesturing, provide a shared language that is a powerful force. Our understanding and knowledge of the benefits of musicking has deepened with the recent advances in neuroscience in combination with the effectiveness of ethnographic studies. Participatory music-making is much more central to our health and success as a species than was thought before, and our experience of rhythm has been clearly identified as a major contributor to wellbeing. Ghanaian recreational music and dance has the capacity to promote and support health and wellness in multi-faceted ways and in varied settings around the world. 


\section{References}

Armstrong, K. (2016). Drumming UP health: A case study of the Carleton West African Rhythm Ensemble. Master's thesis, Carleton University, Ottawa, Canada.

Bernatzky, G., Strickner, S., Presch, M., Wendtner, F., \& Kullich, W. (2012). Music as nonpharmacological pain management in clinics. In R. MacDonald, G. Kreutz, \& L. Mitchell (Eds.), Music, health, and wellbeing (pp. 257-275). Oxford: Oxford University Press.

Burns, J. (2010). Rhythmic archetypes in instrumental music from Africa and the diaspora. Society for Music Theory Online, 16(4). Retrieved from http://www.mtosmt.org/issues/mto.10.16.4/mto.10.16.4.burns.html

Centre for Addiction and Mental Health, Ontario Agency for Health Protection and Promotion (Public Health Ontario), \& Toronto Public Health (2013). Connecting the dots: How Ontario public health units are addressing child and youth mental health. Toronto, ON: Centre for Addiction and Mental Health.

WARE (2012, November 23). Gahu performance by Carleton West African Rhythm Ensemble (WARE) and Baobab Youth Performers [video file]. Recorded by Chris Thompson. Retrieved from https://www.youtube.com/watch?v=WLWNUdn2D6E

Clayton, M. (2012). What is entrainment? Definition and applications in musical research. Empirical Musicology Review, 7(1-2), 49-56.

Clayton, M., Sager, R. \& Will, U. (2004). In time with the music: The concept of entrainment and its significance for ethnomusicology. Counterpoint, 1, 1-82.

Clift, S., \& Hancox, G. (2010). The significance of choral singing for sustaining psychological wellbeing: Findings from a survey of choristers in England, Australia and Germany. Music Performance Research, 3(1), 79-96.

Csikszentmihalyi, M. (1990). Flow: The psychology of optimal experience. New York: Harper Perennial.

Dor, G. (2014). West African drumming and dance in North American universities: An ethnomusicological perspective. Jackson: University Press of Mississippi.

Freeman, J. G., King, M., Pickett, W. (2011). The health of Canada's young people: A mental health focus. Ottawa, Canada: Public Health Agency of Canada.

Friedson, S. (2009). Remains of ritual: Northern gods in a southern land. Chicago: University of Chicago Press.

Keil, C. (1994). Participatory discrepancies and the power of music. In C. Keil \& S. Feld, Music grooves: Essays and dialogues (pp. 96-108). Chicago: University of Chicago Press.

Koen, B., Barz, G., \& Brummel-Smith, K. (2008). Introduction: Confluence of consciousness in music, medicine, and culture. In B. Koen, J. Lloyd, G. Barz, \& K. Brummel-Smith (Eds.), The Oxford handbook of medical ethnomusicology (pp. 3-17). Oxford: Oxford University Press.

LaGasse, A., \& Thaut, M. (2012). Music and rehabilitation: Neurological approaches. In R. MacDonald, G. Kreutz, \& L. Mitchell (Eds.), Music, health, and wellbeing (pp.153163). Oxford: Oxford University Press.

Locke, D. (1987). Drum Gahu: The rhythms of West African drumming. Crown Point, IN: White Cliffs Media.

Locke, D. (2004). The African ensemble in America: Contradictions and possibilities. In T. Solís (Ed.), Performing ethnomusicology: Teaching and representation in world music ensembles (pp. 168-188). Berkeley: University of California Press. 
Phillips-Silver, J., Aktipis, C., \& Bryant, G. (2010). The ecology of entrainment: Foundations of coordinated rhythmic movement. Music Perception, 28(1), 3-14.

Putnam, R. (2000). Bowling alone: The collapse and revival of American community. New York: Simon \& Schuster.

Small, C. (1998). Musicking: The meanings of performing and listening. Hanover: University Press of New England.

Stige, B. (2006). On a notion of participation in music therapy. Nordic Journal of Music Therapy, 15(2), 121-138.

Thaut, M. (2013). Rhythm, music, and the brain: Scientific foundations and clinical applications. New York \& London: Routledge.

Turino, T. (2008). Music as social life: The politics of participation. Chicago: University of Chicago Press.

Will, U. \& Turow, G. (2011) Introduction to entrainment and cognitive ethnomusicology. In J. Berger and G. Turow (Eds.), Music, science and the rhythmic brain: Cultural and clinical implications (pp. 3-30). New York: Routledge. 